\title{
Double Flap Technique is an Effective Reconstruction Procedure After Proximal Gastrectomy for Gastric Cancer
}

\section{Zenichiro Saze ( $\boldsymbol{D}_{\text {z-saze@fmu.ac.jp ) }}$}

Department of Gastrointestinal Tract Surgery, Fukushima Medical University

\section{Koji Kase}

Department of Gastrointestinal Tract Surgery, Fukushima Medical University

\section{Hiroshi Nakano}

Department of Gastrointestinal Tract Surgery, Fukushima Medical University

Naoto Yamauchi

Department of Gastrointestinal Tract Surgery, Fukushima Medical University

\section{Akinao Kaneta}

Department of Gastrointestinal Tract Surgery, Fukushima Medical University

\section{Yohei Watanabe}

Department of Gastrointestinal Tract Surgery, Fukushima Medical University

Hiroyuki Hanayama

Department of Gastrointestinal Tract Surgery, Fukushima Medical University

\section{Suguru Hayase}

Department of Gastrointestinal Tract Surgery, Fukushima Medical University

\section{Tomoyuki Momma}

Department of Gastrointestinal Tract Surgery, Fukushima Medical University

\section{Koji Kono}

Department of Gastrointestinal Tract Surgery, Fukushima Medical University

\section{Research Article}

Keywords: Gastrointestinal, Laparoscopy, Nutrition, Stomach, Oncology

Posted Date: June 14th, 2021

DOI: https://doi.org/10.21203/rs.3.rs-579690/v1

License: (c) (1) This work is licensed under a Creative Commons Attribution 4.0 International License. Read Full License 


\section{Abstract \\ Background}

In accordance with an increase of proximal gastric cancer, proximal gastrectomy came to be widely performed. Several types of reconstructive procedures after proximal gastrectomy have been developed and it is still controversial which procedure has the advantages in preservation of postoperative gastric stump function and nutritional status after proximal gastrectomy. In this study, we retrospectively analyzed reconstructive procedures in our consecutive case series for proximal gastrectomy, with particular focus on postoperative body weight maintenance, nutritional status, and gastric remnant functional preservation.

\section{Methods}

We enrolled 69 patients who received proximal gastrectomy for gastric cancer in our institute from 2005 to 2020. Short-term complications, preservation of gastric remnant functions, nutritional status, and postoperative weight changes were compared.

\section{Results}

After proximal gastrectomy, the numbers of cases receiving Direct Esophago-Gastrostomy, Jejunal Interposition, Double Tract Reconstruction, and Double Flap Technique were 9, 10, 14, and 36, respectively. Double Flap Technique cases suffered no reflux esophagitis after surgery. Prevalence of gastric residual at 12-month after surgery of Double Flap Technique was the lowest. Double Flap Technique group has better tendency in post-operative changes of serum albumin ratios. Furthermore, post-operative body weight changes ratio of Double Flap Technique was the smallest and significantly better than Double Tract at all the time points.

\section{Conclusions}

Double Flap Technique after proximal gastrectomy was considered as the most effective reconstruction which can maintain body weight, cause less reflux esophagitis and gastric residual.

\section{Background}

Even though the number of patients with gastric cancer tends to decrease(1), incidence of proximal gastric cancer is increasing worldwide (2-7). Total gastrectomy had been historically applied for most of gastric cancer which located in upper third of the stomach(8-12), since most of them were advanced cancer when detected, and also poor prognosis $(11,13)$. However, early proximal gastric cancer has been increasingly detected recent years in Japan and Korea $(14,15)$, favorable safety and survival outcome of 
proximal gastrectomy for early proximal gastric cancer was reported recently(14). However, in terms of quality of life after surgery, it was reported that patients who received proximal gastrectomy has frequently incidence of regurgitation and heart burn which causes impairment of quality of life(16-18). To improve this problem, several types of reconstruction procedures after proximal gastrectomy had been developed to prevent regurgitation, such as Jejunal Inter position(19-21), Jejunal Pouch Reconstruction(22, 23), Gastric Tube Reconstruction(24), Esophago-Gastrostomy With Fundoplication(18, 25), and Double Tract Reconstruction(26-29). Particularly, Double Flap Technique (30-32) as the reconstructive procedure which can strongly prevent regurgitation after proximal gastrectomy was firstly introduced in 2001 by Kamikawa et. al (30). Double Flap Technique is the esophago-gastrostomy procedure, which can strongly prevent regurgitation by developing "new cardia" as a result of buried esophagus in the anterior gastric wall by sero-muscular flaps. However, it is unclear whether Double Flap Technique has advantages in terms of postoperative nutritional status and postoperative gastric remnant functional preservation as long-term effect. Therefore, we retrospectively made a comparative analysis for reconstructive procedures after proximal gastrectomy for gastric cancer in this study.

\section{Methods}

\section{Patients}

Patients with gastric cancers who underwent proximal gastrectomy from January 2005 to June 2020 at the Department of Gastrointestinal Tract Surgery, Fukushima Medical University Hospital were enrolled in the study. All cases with gastric cancers were preoperatively diagnosed at our institution. Tumors of all cases were located in upper third of the stomach, and those were suggested that at least one-half of the stomach could be preserved preoperatively. Patients who underwent proximal gastrectomy with lower esophageal resection and intra-mediastinal anastomosis were excluded in the present study. The clinical and pathological data were retrospectively collected from medical records, with the last follow-up in Jan 2021. These data included age, gender, body weight, hematological examination, tumor location, tumor depth, lymph nodes metastasis, and TNM classification (8th edition). Treatment was performed after obtaining informed consent.

\section{Surgical procedures of proximal gastrectomy and reconstructive procedures}

Proximal gastrectomy was performed under open abdominal surgery, hand assisted laparoscopic surgery (HALS), laparoscopic surgery, or robotic assisted laparoscopic surgery. D1 or D1 + lymph node dissection according to the Japanese gastric cancer treatment guidelines 2018 (5th edition)(33) was performed. Reconstruction was performed by Direct Esophago-Gastrostomy (DEG), Jejunal Interposition (JIP), Double Tract Reconstruction (DTR), or Double Flap Technique (DFT) by physician's choice. Each reconstruction procedure is summarized as follows. DEG: Esophago-gastrostomy was performed by circular stapler inserted from small incision of anterior wall in gastric remnant, then incision was closed by hand-stitch using absorbable suture thread. Fundoplication was added to some cases of them. JIP: 
The divided jejunum was brought up via the retro-colic route and anastomosed side-to-end with the esophagus using circular stapler and end-to-side with the remaining stomach by hand-stitch. DTR: Esophago-jejunostomy and jejuno-gastrostomy were anastomosed side-by-side by linear stapler, then, entry hole of stapler was closed by hand-sewn. DFT: Gastric sero-muscular flaps were prepared extracorporeally. Then, posterior wall of full-thickness esophagus and gastric mucosa were sewn by running suture, and anterior wall was sewn by layer to layer running suture or Gambee's interrupted suture. Finally, esophago-gastrostomy was wrapped by bilateral gastric sero-muscular flaps.

\section{Evaluation Method}

After surgery, patients were followed up at outpatient clinic every 3 months for the first and 2nd year and every 6 months thereafter until 5 years after surgery. Body weight, albumin, hemoglobin, and lymphocyte count at 3-month, 6-month, and 12-month post-surgery were evaluated. Endoscopic examination was performed annually, however, when anastomotic stenosis was suspected, an endoscopic examination was performed and bougienage therapy was applied if necessary.

\section{Statistical Analysis}

Data were analyzed using the SPSS statistical software program version 27.0 for Mac (SPSS Inc., Chicago, III., USA). Continuous variables were analyzed using Student's t test (2-sided test). The $\chi 2$ test with the Yates' correction for $2 \times 2$ tables were used to compare categorized data. The one-way analysis of variance (ANOVA) is used to determine whether there are any statistically significant differences between the means of three or more independent groups and the Bonferroni correction was used as posthoc analysis. In addition, multivariate binary logistic regression analysis with corresponding odds ratios (OR) and 95\% confidence intervals (Cl) was performed to identify independent risk factors for postoperative weight loss rate above $12 \%$ at 12 -month after surgery. $p<0.05$ was considered statistically significant.

\section{Results}

\section{Patient demographics}

Sixty-nine patients who underwent proximal gastrectomy were enrolled. The mean age of the patients was 70.0 (range 43-94) years old and there were 53 men (76.8\%). Fourteen patients had advanced cancer (pT1a, 11; pT1b, 44; pT2, 9; pT3, 3; pT4a, 2), although all cases were pre-operatively diagnosed as early gastric cancer. Nodal metastases were observed in five patients; (N1, 4; N2, 1). No distant metastasis was observed. All operations were undertaken with curative intent. Nineteen cases underwent open proximal gastrectomy, 1 case underwent hand-assisted laparoscopic proximal gastrectomy, 36 cases had laparoscopic proximal gastrectomy, and 13 cases had robot assisted proximal gastrectomy, respectively. Decisions on which approach was done was by physician's choice. Patient background is shown in Table 1 and there was no statistically significant difference between each group preoperatively. 
Table 2 shows surgical background and post-operative courses of each reconstruction. Frequency of D1+ lymph node dissection in DFT group was much higher than other groups. Most of cases of DEG and JIP were performed by open laparotomy, in contrast, all cases of DTR were laparoscopic surgery, and robotic surgery was performed in only DFT group. Pathological depth of tumor invasion, lymph node metastasis, and pathological stage were not significantly different between groups. Hospital stay of DFT was the shortest and significantly shorter than DEG and JIP ( $p<0.001, p=0.014$, data is not shown in Table 2). Post-operative short-term complications including anastomotic leakage and pancreatic fistula were not significantly different between each group. No surgical death was observed in each reconstruction group. Comparison of long-term complications shows no significant difference in the rate of anastomotic stenosis as shown in Table 2. Although ratios of suffering reflux gastritis above grade A in Los Angeles classification after surgery between each reconstruction group were not significantly different, only DFT cases had no reflux esophagitis. And DFT group is the least frequently of gastric residual in according to observations of post-operative upper gastrointestinal endoscope (Table 2).

\section{Indicators of post-operative nutritional status changes}

Table 3 shows post-operative indicators of nutritional status changes. Changes of serum albumin ratios at 6-month and 12-month showed a significant difference between the reconstructive groups and DFT group has better tendency in serum albumin ratios. Hemoglobin ratios, and lymphocyte count ratios were not significantly different between each group.

\section{Post-operative body weight changes}

Figure 1 shows body weight changes after proximal gastrectomy of each reconstruction group. The oneway ANOVA detected significant differences between groups at 3-month, 6-month, and 12-month after surgery $(p=0.001,0.002$, and 0.024 , respectively) and DFT group shows the most favorable results. Furthermore, multiple comparisons showed that body weight loss ratio of DFT was significantly better than DTR at all the time point ( $\mathrm{p}=0.001,0.003$, and 0.014 , respectively). Furthermore, multivariate analysis revealed that reconstruction with other than DFT was independent risk factor for post-operative weight loss rate above $12 \%$ at 12 -month after surgery (Table 4 ).

\section{Discussion}

In this study, we showed DFT has the advantages in less body weight loss, less reflex esophagitis, and less gastric residual after proximal gastrectomy. In the past, total gastrectomy was recommended rather than proximal gastrectomy for upper gastric cancers, since that the most of upper gastric cancers were advanced at the time of diagnosis and prognosis of this population was very poor(8-12). Also, proximal gastrectomy was not recommended in terms of postoperative morbidity and quality of life (QOL), since frequency of reflux esophagitis and heartburn after proximal gastrectomy were significantly higher than those after total gastrectomy, and no benefit was observed in the point of body weight loss compared with total gastrectomy $(16,17,34)$. Furthermore, it was reported that QOL after proximal gastrectomy is worse than total gastrectomy because of high frequency of nausea and vomiting(17). However, most of 
reconstructive procedures after proximal gastrectomy in these previous studies were esophagogastrostomy without any anti-reflex mechanisms. Recently, it has been reported that prognosis after proximal gastrectomy is oncologically similar with that of total gastrectomy $(34,35)$, and it was showed that proximal gastrectomy with supra-pancreatic lymph nodes dissection has favorable long-term outcome in Japan(14). As a result, the number of proximal gastrectomy has been increasing in Japan. In addition, nutritional benefit of proximal gastrectomy compared with total gastrectomy was recently reported $(27-29,36)$. Furthermore, several types of reconstructive procedures which can prevent regurgitation, such as JIP(19-21), jejunal pouch reconstruction(22, 23), gastric tube reconstruction(24), DEG with fundoplication(18, 25), DTR(26-29), and DFT (30) (31) (32), were developed. However, it was not clear which reconstructive procedure has advantages of postoperative QOL. In this study, we retrospectively analyzed our consecutive case series for proximal gastrectomy, with particular focus on postoperative body weight maintenance, nutritional status, and gastric remnant functional preservation. In this study, we showed that DFT is the most effective reconstructive procedure to prevent reflux esophagitis, since no reflux esophagitis was observed in DFT group and reconstruction with anti-reflux procedure other than DFT has some cases of reflux esophagitis.

Also, we showed that the rate of anastomotic stenosis after DFT (8.3\%) was less frequent in comparison to other reconstruction procedures. However, it should be careful in interpreting of this anastomotic stenosis date. It is generally accepted that circular stapler is widely used for esophago-jejunostomy(3739) and the stricture rate with a stapler anastomosis was reported to be high compared to hand-sewn anastomosis(40-42). Since esophago-gastrostomy in DFT is performed by hand-sewn, it makes anastomosis soft and flexible, and can prevents the anastomotic stenosis(43). In other words, the less anastomotic stenosis rate in DFT may be due to hand-sewn anastomosis, not by DFT procedure itself. The rate of anastomotic stenosis after DFT has been reported to be $5.5 \mathrm{-1}-9 \mathrm{Q}(32,43,44)$ and $8.3 \%$ in the present study. Therefore, we still need to improve and modify a DFT procedure which can prevent anastomotic stenosis more completely. Now, we are employing Gambee's method for suturing anterior wall of esophago-gastrostomy in the DFT reconstruction, instead of layer-to-layer running suture. Moreover, there are some reports that DFT was performed by laparoscopic surgery, it may have more benefit due to the minimally invasive surgery $(32,36)$. However, laparoscopic DFT is cumbersome due to its restriction of movement, surgeons need to be an expert in laparoscopic suturing skill. However, it may be resolved by robotic surgery(45). In this study, 13 cases of DFT were performed by robotic assisted surgery and we have good achievement in robotic DFT.

In this study, we showed that post-operative body weight loss after Double Flap Technique is the best outcome. We believe that one possible reason to improve body weight loss in DFT is less regurgitation and less gastric residual in comparison to other procedures. Unfortunately, we were not able to show solid advantage of DFT in postoperative nutritional status within hematological examination compared to other reconstructive procedures, although there was minor advantage in DFT group for albumin change.

Although the present study has provided some important information for clinical practice, it has some limitations. In particular, this was a retrospective study with a small sample size at a single institution. 
Further accumulation of cases is required. Second, the study may have bias, because we did not evaluate the size of remnant stomach, which may affect to post-operative body weight loss and nutritional status.

\section{Conclusions}

The advantages of DFT after proximal gastrectomy for gastric cancer was demonstrated. DFT markedly decreased risk of post-operative body weight loss, reflux esophagitis, and gastric residual in comparison with other reconstructive procedures for proximal gastrectomy.

\section{Abbreviations}

HALS; hand assisted laparoscopic surgery

DEG; Direct Esophago-Gastrostomy

JIP; Jejunal Interposition

DTR; Double Tract Reconstruction

DFT; Double Flap Technique

ANOVA; analysis of variance

OR; odds ratios

QOL; quality of life

\section{Declarations}

Ethics approval and consent to participate

The study was conducted in accordance with the Declaration of Helsinki and was approved by the Institutional Review Board of Fukushima Medical University. (approval number: 30127)

Consent for publication

Not applicable

Availability of data and materials

The datasets used and/or analysed during the current study are available from the corresponding author on reasonable request.

Competing interests 
The authors declare that they have no competing interests.

Funding

This research did not receive any specific grant from funding agencies in the public, commercial, or notfor-profit sectors.

Authors' contributions

ZS was a major contributor in writing the manuscript. ZS, KK, HN, NY, AK, YW, HH, SH, and TM were involved in study design and data interpretation and performed operations. ZS and KK were involved in the data analysis. All authors read and approved the final manuscript.

Acknowledgements

Not applicable

\section{References}

1. Matsuno K, Ishihara R, Ohmori M, Iwagami H, Shichijyo S, Maekawa A, et al. Time trends in the incidence of esophageal adenocarcinoma, gastric adenocarcinoma, and superficial esophagogastric junction adenocarcinoma. Journal of gastroenterology. 2019.

2. Borch K, Jönsson B, Tarpila E, Franzén T, Berglund J, Kullman E, et al. Changing pattern of histological type, location, stage and outcome of surgical treatment of gastric carcinoma. British Journal of Surgery. 2000;87(5):618-26.

3. Deans C, Yeo MS, Soe MY, Shabbir A, Ti TK, So JB. Cancer of the gastric cardia is rising in incidence in an Asian population and is associated with adverse outcome. World journal of surgery. 2011;35(3):617-24.

4. Carr JS, Zafar SF, Saba N, Khuri FR, El-Rayes BF. Risk factors for rising incidence of esophageal and gastric cardia adenocarcinoma. Journal of Gastrointestinal Cancer. 2013;44(2):143-51.

5. Brown LM, Devesa SS. Epidemiologic trends in esophageal and gastric cancer in the United States. Surgical Oncology Clinics of North America. 2002;11(2):235-56.

6. Blot WJ, Devesa SS, Kneller RW, Fraumeni JF. Rising Incidence of Adenocarcinoma of the Esophagus and nGastric Cardia. JAMA: The Journal of the American Medical Association. 1991;265(10):1287-9.

7. Powell J, McConkey CC. The rising trend in oesophageal adenocarcinoma and gastric cardia. European Journal of Cancer Prevention. 1992;1(3):265-9.

8. Papachristou DN, Fortner JG. Adenocarcinoma of the gastric cardia. The choice of gastrectomy. Annals of surgery. 1980;192(1):58-64.

9. Siewert JR, Böttcher K, Stein HJ, Roder JD, Busch R. Problem of proximal third gastric carcinoma. World journal of surgery. 1995;19(4):523-31. 
10. Stipa S, Di Giorgio A, Ferri M. Surgical treatment of adenocarcinoma of the cardia. Surgery. 1992;111(4):386-93.

11. Harrison LE, Karpeh MS, Brennan MF. Proximal gastric cancers resected via a transabdominal-only approach: Results and comparisons to distal adenocarcinoma of the stomach. Annals of surgery. 1997;225(6):678-85.

12. Kim JH, Park SS, Kim J, Boo YJ, Kim SJ, Mok YJ, et al. Surgical outcomes for gastric cancer in the upper third of the stomach. World journal of surgery. 2006;30(10):1870-6.

13. Salvon-Harman JC, Cady B, Nikulasson S, Khettry U, Stone MD, Lavin P. Shifting Proportions of Gastric Adenocarcinomas. Archives of Surgery. 1994;129(4):381-9.

14. Katai H, Sano T, Fukagawa T, Shinohara H, Sasako M. Prospective study of proximal gastrectomy for early gastric cancer in the upper third of the stomach. The British journal of surgery. 2003;90(7):8503.

15. Korean Gastric Cancer Association Nationwide Survey on Gastric Cancer in 2014. J Gastric Cancer. 2016;16(3):131-40.

16. Buhl K, Schlag P, Herfarth C. Quality of life and functional results following different types of resection for gastic carcinoma. European Journal of Surgical Oncology. 1990;16(4):404-9.

17. Karanicolas PJ, Graham D, Gönen M, Strong VE, Brennan MF, Coit DG. Quality of life after gastrectomy for adenocarcinoma: A prospective cohort study. Annals of surgery. 2013;257(6):103946.

18. Nakamura $M$, Nakamori $M$, Ojima T, Katsuda $M$, lida $T$, Hayata $K$, et al. Reconstruction after proximal gastrectomy for early gastric cancer in the upper third of the stomach: an analysis of our 13-year experience. Surgery. 2014;156(1):57-63.

19. Takeshita K, Saito N, Saeki I, Honda T, Tani M, Kando F, et al. Proximal gastrectomy and jejunal pouch interposition for the treatment of early cancer in the upper third of the stomach: Surgical techniques and evaluation of postoperative function. Surgery. 1997;121(3):278-86.

20. Tokunaga M, Ohyama S, Hiki N, Hoshino E, Nunobe S, Fukunaga T, et al. Endoscopic evaluation of reflux esophagitis after proximal gastrectomy: Comparison between esophagogastric anastomosis and jejunal interposition. World journal of surgery. 2008;32(7):1473-7.

21. Nozaki I, Hato S, Kobatake T, Ohta K, Kubo Y, Kurita A. Long-term outcome after proximal gastrectomy with jejunal interposition for gastric cancer compared with total gastrectomy. World journal of surgery. 2013;37(3):558-64.

22. Chang HY, Byung HS, Won KH, Won KP. Proximal gastrectomy reconstructed by jejunal pouch interposition for upper third gastric cancer: Prospective randomized study. World journal of surgery. 2005;29(12):1592-9.

23. Iwata T, Kurita N, Ikemoto T, Nishioka M, Andoh T, Shimada M. Evaluation of reconstruction after proximal gastrectomy: prospective comparative study of jejunal interposition and jejunal pouch interposition. Hepatogastroenterology. 2006;53(68):301-3. 
24. Shiraishi N, Adachi Y, Kitano S, Kakisako K, Inomata M, Yasuda K. Clinical outcome of proximal versus total gastrectomy for proximal gastric cancer. World journal of surgery. 2002;26(9):1150-4.

25. Sakuramoto S, Yamashita K, Kikuchi S, Futawatari N, Katada N, Moriya H, et al. Clinical Experience of Laparoscopy-Assisted Proximal Gastrectomy with Toupet-Like Partial Fundoplication in Early Gastric Cancer for Preventing Reflux Esophagitis. Journal of the American College of Surgeons. 2009;209(3):344-51.

26. Aikou T, Natsugoe S, Shimazu H, Nishi M. Antrum preserving double tract method for reconstruction following proximal gastrectomy. The Japanese Journal of Surgery. 1988;18(1):114-5.

27. Ahn SH, Jung DH, Son SY, Lee CM, Park DJ, Kim HH. Laparoscopic double-tract proximal gastrectomy for proximal early gastric cancer. Gastric Cancer. 2014;17(3):562-70.

28. Nomura E, Lee SW, Kawai M, Yamazaki M, Nabeshima K, Nakamura K, et al. Functional outcomes by reconstruction technique following laparoscopic proximal gastrectomy for gastric cancer: Double tract versus jejunal interposition. World journal of surgical oncology. 2014;12(1).

29. Jung DH, Lee Y, Kim DW, Park YS, Ahn SH, Park DJ, et al. Laparoscopic proximal gastrectomy with double tract reconstruction is superior to laparoscopic total gastrectomy for proximal early gastric cancer. Surgical Endoscopy. 2017;31(10):3961-9.

30. Kamikawa Y KT, Kamiyama $S$ et al. A new procedure of esophagogastrostomy to prevent reflux following proximal gastrectomy (in Japanese). Shoukakigeka. 2001;24:1053-60.

31. Mine S, Nunobe S, Watanabe M. A Novel Technique of Anti-reflux Esophagogastrostomy Following Left Thoracoabdominal Esophagectomy for Carcinoma of the Esophagogastric Junction. World journal of surgery. 2015;39(9):2359-61.

32. Shoji Y, Nunobe S, Ida S, Kumagai K, Ohashi M, Sano T, et al. Surgical outcomes and risk assessment for anastomotic complications after laparoscopic proximal gastrectomy with double-flap technique for upper-third gastric cancer. Gastric Cancer. 2019.

33. Japanese Gastric Cancer A. Japanese gastric cancer treatment guidelines 2018 (5th edition). Gastric Cancer. 2020.

34. An JY, Youn HG, Choi MG, Noh JH, Sohn TS, Kim S. The difficult choice between total and proximal gastrectomy in proximal early gastric cancer. American journal of surgery. 2008;196(4):587-91.

35. Harrison LE, Karpeh MS, Brennan MF. Total gastrectomy is not necessary for proximal gastric cancer. Surgery. 1998;123(2):127-30.

36. Hayami M, Hiki N, Nunobe S, Mine S, Ohashi M, Kumagai K, et al. Clinical Outcomes and Evaluation of Laparoscopic Proximal Gastrectomy with Double-Flap Technique for Early Gastric Cancer in the Upper Third of the Stomach. Annals of Surgical Oncology. 2017;24(6):1635-42.

37. Jung YJ, Kim DJ, Lee JH, Kim W. Safety of intracorporeal circular stapling esophagojejunostomy using trans-orally inserted anvil (OrVil ${ }^{\mathrm{TM}}$ ) following laparoscopic total or proximal gastrectomy comparison with extracorporeal anastomosis. World journal of surgical oncology. 2013;11.

38. Hirahara N, Monma H, Shimojo Y, Matsubara T, Hyakudomi R, Yano S, et al. Reconstruction of the esophagojejunostomy by double stapling method using $\mathrm{EEA}^{\mathrm{TM}} \mathrm{OrVil}^{\mathrm{TM}}$ in laparoscopic total 
gastrectomy and proximal gastrectomy. World journal of surgical oncology. 2011;9.

39. Hiki N, Fukunaga T, Yamaguchi T, Nunobe S, Tokunaga M, Ohyama S, et al. Laparoscopic esophagogastric circular stapled anastomosis: A modified technique to protect the esophagus. Gastric Cancer. 2007;10(3):181-6.

40. Wong J, Cheung H, Lui R, Fan YW, Smith A, Siu KF. Esophagogastric anastomosis performed with a stapler: the occurrence of leakage and stricture. Surgery. 1987;101(4):408-15.

41. Tokunaga Y, Ryo J, Kitaoka A, Yagi T, Tokuka A, Ohsumi K. Jejunal pouch to avoid stricture after esophagojejunostomy with circular stapler. Journal of the American College of Surgeons. 1999;189(5):466-9.

42. Takeyoshi I, Ohwada S, Ogawa T, Kawashima Y, Ohya T, Kawate S, et al. Esophageal anastomosis following gastrectomy for gastric cancer: Comparison of hand-sewn and stapling technique. HepatoGastroenterology. 2000;47(34):1026-9.

43. Kuroda S, Nishizaki M, Kikuchi S, Noma K, Tanabe S, Kagawa S, et al. Double-Flap Technique as an Antireflux Procedure in Esophagogastrostomy after Proximal Gastrectomy. Journal of the American College of Surgeons. 2016;223(2):e7-e13.

44. Kuroda S, Choda Y, Otsuka S, Ueyama S, Tanaka N, Muraoka A, et al. Multicenter retrospective study to evaluate the efficacy and safety of the double-flap technique as antireflux esophagogastrostomy after proximal gastrectomy (rD-FLAP Study). Annals of gastroenterological surgery. 2019;3(1):96103.

45. Shibasaki S, Suda K, Nakauchi M, Kikuchi K, Kadoya S, Ishida Y, et al. Robotic valvuloplastic esophagogastrostomy using double flap technique following proximal gastrectomy: technical aspects and short-term outcomes. Surgical Endoscopy. 2017;31(10):4283-97.

\section{Tables}

Table 1 Preoperative patient demographics

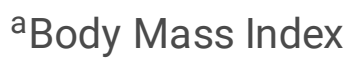

There was no statistically significant difference between each group preoperatively. 


\begin{tabular}{|llll|}
\hline Variables & OR & $95 \% \mathrm{Cl}$ & p value \\
\hline Age $<70$ & 0.536 & $0.148-1.943$ & 0.343 \\
\hline Male & 1.884 & $0.376-9.452$ & 0.441 \\
\hline Pre-operative BMI ${ }^{\mathrm{a}}<22$ & 2.925 & $0.724-11.823$ & 0.132 \\
\hline Laparotomy and HALS & 4.319 & $0.708-26.355$ & 0.113 \\
\hline D1+ Lymph node dissection & 2.85 & $0.456-17.816$ & 0.263 \\
\hline Short-term complication ${ }^{d}$ & 4.484 & $0.274-73.37$ & 0.293 \\
\hline Reconstruction with other than DFT & 6.037 & $1.226-29.732$ & $0.027 *$ \\
\hline
\end{tabular}

Table 2 Operative results and post-operative courses 


\begin{tabular}{|c|c|c|c|c|c|}
\hline & $\begin{array}{l}\text { DEG } \\
(\mathrm{N}=9)\end{array}$ & $\begin{array}{l}\text { JIP } \\
(N=10)\end{array}$ & $\begin{array}{l}\text { DTR } \\
(\mathrm{N}=14)\end{array}$ & $\begin{array}{l}\text { DFT } \\
(\mathrm{N}=36)\end{array}$ & $\mathrm{p}$-value \\
\hline \multicolumn{6}{|l|}{ Extent of dissection (\%) } \\
\hline D1 & $5(55.6 \%)$ & $4(40.0 \%)$ & $4(28.6 \%)$ & $3(8.3 \%)$ & $<0.001^{*}$ \\
\hline D1+ & $4(44.4 \%)$ & $6(60.0 \%)$ & $\begin{array}{l}10 \\
(71.4 \%)\end{array}$ & $\begin{array}{l}33 \\
(91.7 \%)\end{array}$ & \\
\hline \multicolumn{6}{|l|}{ Approach } \\
\hline Open laparotomy & $6(66.7 \%)$ & $10(100 \%)$ & $0(0 \%)$ & $3(8.3 \%)$ & $<0.001^{\star}$ \\
\hline HALS & $1(11.1 \%)$ & $0(0 \%)$ & $0(0 \%)$ & $0(0 \%)$ & \\
\hline Laparoscopy & $2(22.2 \%)$ & $0(0 \%)$ & $14(100 \%)$ & $\begin{array}{l}20 \\
(55.6 \%)\end{array}$ & \\
\hline Robotic laparoscopy & $0(0 \%)$ & $0(0 \%)$ & $0(0 \%)$ & $\begin{array}{l}13 \\
(36.1 \%)\end{array}$ & \\
\hline \multicolumn{6}{|l|}{ Depth of invasion (\%) } \\
\hline $\mathrm{T} 1 \mathrm{a}$ & $2(22.2 \%)$ & $0(0 \%)$ & $4(28.6 \%)$ & $5(13.9 \%)$ & 0.446 \\
\hline $\mathrm{T} 1 \mathrm{~b}$ & $5(55.6 \%)$ & $9(90.0 \%)$ & $8(57.1 \%)$ & $\begin{array}{l}22 \\
(61.1 \%)\end{array}$ & \\
\hline T2 & $0(0 \%)$ & $1(10.0 \%)$ & $1(7.1 \%)$ & $7(19.4 \%)$ & \\
\hline T3 & $1(11.1 \%)$ & $0(0 \%)$ & $1(7.1 \%)$ & $1(2.8 \%)$ & \\
\hline $\mathrm{T} 4 \mathrm{a}$ & $1(11.1 \%)$ & $0(0 \%)$ & $0(0 \%)$ & $1(2.8 \%)$ & \\
\hline \multicolumn{6}{|l|}{ Lymph node metastasis } \\
\hline NO & $\begin{array}{l}9 \\
(100.0 \%)\end{array}$ & $\begin{array}{l}10 \\
(100.0 \%)\end{array}$ & $\begin{array}{l}12 \\
(85.7 \%)\end{array}$ & $\begin{array}{l}33 \\
(91.7 \%)\end{array}$ & 0.462 \\
\hline N1 & $0(0 \%)$ & $0(0 \%)$ & $1(7.1 \%)$ & $3(8.3 \%)$ & \\
\hline N2 & $0(0 \%)$ & $0(0 \%)$ & $1(7.1 \%)$ & $0(0 \%)$ & \\
\hline \multicolumn{6}{|l|}{ Pathological Stage $\triangle U I C C$ 7th $\rrbracket$} \\
\hline 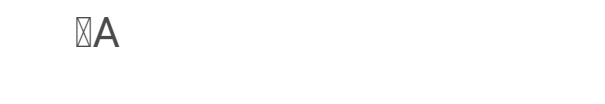 & $7(77.8 \%)$ & $9(90.0 \%)$ & $11(78.6 \%)$ & $\begin{array}{l}26 \\
(72.2 \%)\end{array}$ & 0.590 \\
\hline IB & $0(0 \%)$ & $1(10.0 \%)$ & $1(7.1 \%)$ & $8(22.2 \%)$ & \\
\hline$\nabla \mathrm{A}$ & $1(11.1 \%)$ & $0(0 \%)$ & $1(7.1 \%)$ & $1(2.8 \%)$ & \\
\hline$\bowtie \mathrm{B}$ & $1(11.1 \%)$ & $0(0 \%)$ & $1(7.1 \%)$ & $1(2.8 \%)$ & \\
\hline Hospital stay (days, mean $\pm S D$ ) & $18.1 \pm 5.8$ & $15.2 \pm 5.6$ & $12.2 \pm 3.6$ & $10.5 \pm 3.3$ & $<0.001^{*}$ \\
\hline
\end{tabular}


Short-term complications

$C D^{\mathrm{a}}$ grade $\otimes$

\begin{tabular}{|c|c|c|c|c|c|}
\hline Ileus & $0(0 \%)$ & $0(0 \%)$ & $1(7.1 \%)$ & $3(8.3 \%)$ & 0.650 \\
\hline Pneumonia & $0(0 \%)$ & $0(0 \%)$ & $1(7.1 \%)$ & $0(0 \%)$ & 0.263 \\
\hline (\%) Postoperative hemorrhage & $1(11.1 \%)$ & $1(10 \%)$ & $0(0 \%)$ & $1(2.8 \%)$ & 0.456 \\
\hline Anastomotic leakage & $0(0 \%)$ & $0(0 \%)$ & $0(0 \%)$ & $1(2.8 \%)$ & 0.818 \\
\hline Pancreatic fistula & $0(0 \%)$ & $0(0 \%)$ & $1(7.1 \%)$ & $1(2.8 \%)$ & 0.690 \\
\hline \multicolumn{6}{|l|}{ Long-term complications } \\
\hline Anastomotic stenosis $^{\mathrm{b}}$ & $1(11.1 \%)$ & $1(10 \%)$ & $3(21.4 \%)$ & $3(8.3 \%)$ & 0.935 \\
\hline Reflux esophagitis ${ }^{c}$ & $2(22.2 \%)$ & $1(10 \%)$ & $3(21.4 \%)$ & $0(0 \%)$ & 0.203 \\
\hline Gastric residual $^{\mathrm{d}}$ & $3(33.3 \%)$ & $7(70 \%)$ & $2(14.3 \%)$ & $5(13.9 \%)$ & $0.017 *$ \\
\hline
\end{tabular}

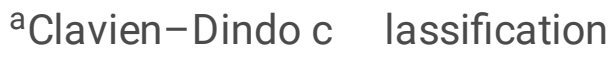

${ }^{b}$ Anastomotic stenosis which required balloon dilatation

${ }^{\mathrm{C}}$ Reflux esophagitis grade $\geq \mathrm{A}$ in Los Angeles classification

${ }^{\mathrm{d}}$ Gastric residual observed by endoscopy at 1 year after surgery

*Statistically significant

Hospital stay of DFT was the shortest and significantly shorter than DEG and JIP. Comparison of longterm complications shows no significant difference in the rate of anastomotic stenosis. DFT cases had no reflux esophagitis. DFT group is the least frequently of gastric residual.

Table 3 Post-operative indicators of nutritional status changes 


\begin{tabular}{|c|c|c|c|c|c|c|}
\hline & period & $\begin{array}{l}\mathrm{DEG} \\
(\mathrm{N}=9)\end{array}$ & $\mathrm{JIP}(\mathrm{N}=10)$ & $\begin{array}{l}\text { DTR } \\
(\mathrm{N}=14)\end{array}$ & $\begin{array}{l}\text { DFT } \\
(\mathrm{N}=36)\end{array}$ & $\mathrm{p}$-value \\
\hline \multirow[t]{3}{*}{ Albumin change } & $3 m$ & $89.5 \pm 8.1$ & $98.0 \pm 10.4$ & $92.7 \pm 6.7$ & $97.2 \pm 9.4$ & 0.134 \\
\hline & $6 m$ & $89 \pm 9.9$ & $103.1 \pm 10.2$ & $93.9 \pm 11.0$ & $100.1 \pm 11.6$ & $0.041^{*}$ \\
\hline & $12 m$ & $94.2 \pm 5.5$ & $106.8 \pm 12.2$ & $97.4 \pm 5.3$ & $100.2 \pm 9.1$ & $0.037^{*}$ \\
\hline \multirow[t]{3}{*}{ Hemoglobin change } & $3 m$ & $94.3 \pm 6.4$ & $90.7 \pm 9.2$ & $91.43 \pm 6.6$ & $103.9 \pm 48.9$ & 0.655 \\
\hline & $6 m$ & $88.4 \pm 3.2$ & $89.7 \pm 10.6$ & $92.7 \pm 6.2$ & $105.5 \pm 54.3$ & 0.636 \\
\hline & $12 m$ & $94.8 \pm 7.0$ & $92.4 \pm 8.6$ & $92.5 \pm 5.8$ & $98.2 \pm 9.5$ & 0.172 \\
\hline \multirow{3}{*}{$\begin{array}{l}\text { Lymphocyte count } \\
\text { change }\end{array}$} & $3 m$ & $69.6 \pm 14.0$ & $88.5 \pm 28.6$ & $87 \pm 28.3$ & $118.1 \pm 51.0$ & 0.107 \\
\hline & $6 m$ & $61.6 \pm 39.1$ & $79.8 \pm 25.4$ & $90.8 \pm 26.1$ & $121.8 \pm 69.6$ & 0.183 \\
\hline & $12 m$ & $67.2 \pm 4.6$ & $100.8 \pm 48.1$ & $103 \pm 39.0$ & $125.2 \pm 88.9$ & 0.583 \\
\hline
\end{tabular}

Values are percentage of post-operative to the pre-operative (mean $\pm S D$ )

*Statistically significant

Changes of serum albumin ratios showed DFT group has better tendency in serum albumin ratios. Hemoglobin ratios, and lymphocyte count ratios were not significantly different between each group.

Table 4 Multivariate analysis for risk of post-operative weight loss rate above $12 \%$ at 1 -year after surgery

\begin{tabular}{|c|c|c|c|c|c|}
\hline & $\begin{array}{l}\text { DEG } \\
(\mathrm{N}=9)\end{array}$ & $\begin{array}{l}\text { JIP } \\
(N=10)\end{array}$ & $\begin{array}{l}\text { DTR } \\
(\mathrm{N}=14)\end{array}$ & $\begin{array}{l}\text { DFT } \\
(\mathrm{N}=36)\end{array}$ & p-value \\
\hline Age, y, median (mean $\pm S D)$ & $75.7 \pm 9.8$ & $69.8 \pm 8.4$ & $66.8 \pm 12.9$ & $69.9 \pm 8.1$ & 0.196 \\
\hline 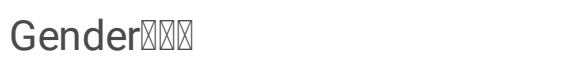 & & & & & 0.473 \\
\hline Male & $8(88.9 \%)$ & $8(80.0 \%)$ & $\begin{array}{l}12 \\
(85.7 \%)\end{array}$ & $\begin{array}{l}25 \\
(69.4 \%)\end{array}$ & \\
\hline Female & $1(11.1 \%)$ & $2(20.0 \%)$ & $2(14.3 \%)$ & $\begin{array}{l}11 \\
(30.6 \%)\end{array}$ & \\
\hline $\mathrm{BMI}^{\mathrm{a}}, \mathrm{kg} / \mathrm{m} 2($ mean $\pm \mathrm{SD})$ & $23.7 \pm 3.6$ & $22.9 \pm 3.5$ & $24.2 \pm 4.2$ & $23.1 \pm 3.5$ & 0.764 \\
\hline Albumin, g/dl (mean $\pm S D)$ & $4.0 \pm 0.35$ & $3.95 \pm 0.38$ & $4.3 \pm 0.36$ & $4.0 \pm 0.39$ & 0.084 \\
\hline Hemoglobin, g/dl (mean $\pm S D)$ & $12.9 \pm 1.4$ & $13.0 \pm 1.1$ & $13.3 \pm 1.7$ & $12.8 \pm 2.1$ & 0.852 \\
\hline $\begin{array}{l}\text { Lymphocytes count, / } \mu \mathrm{L} \\
\text { (mean } \pm \text { SD) }\end{array}$ & $1755 \pm 638$ & $1771 \pm 351$ & $1719 \pm 539$ & $1481 \pm 476$ & 0.235 \\
\hline
\end{tabular}


a Body Mass Index

bHand Assisted Laparoscopy

${ }^{\mathrm{C}}$ Double Flap Technique

${ }^{\mathrm{d} C o m p l i c a t i o n s ~ g r a d e ~ I I ~ o r ~ a b o v e ~ i n ~ C l a v i e n-D i n d o ~ c l a s s i f i c a t i o n ~}$

*Statistically significant

Multivariate analysis revealed that reconstruction with other than DFT was independent risk factor for post-operative weight loss rate above $12 \%$ at 12 -month after surgery.

Figures 
Fig.1

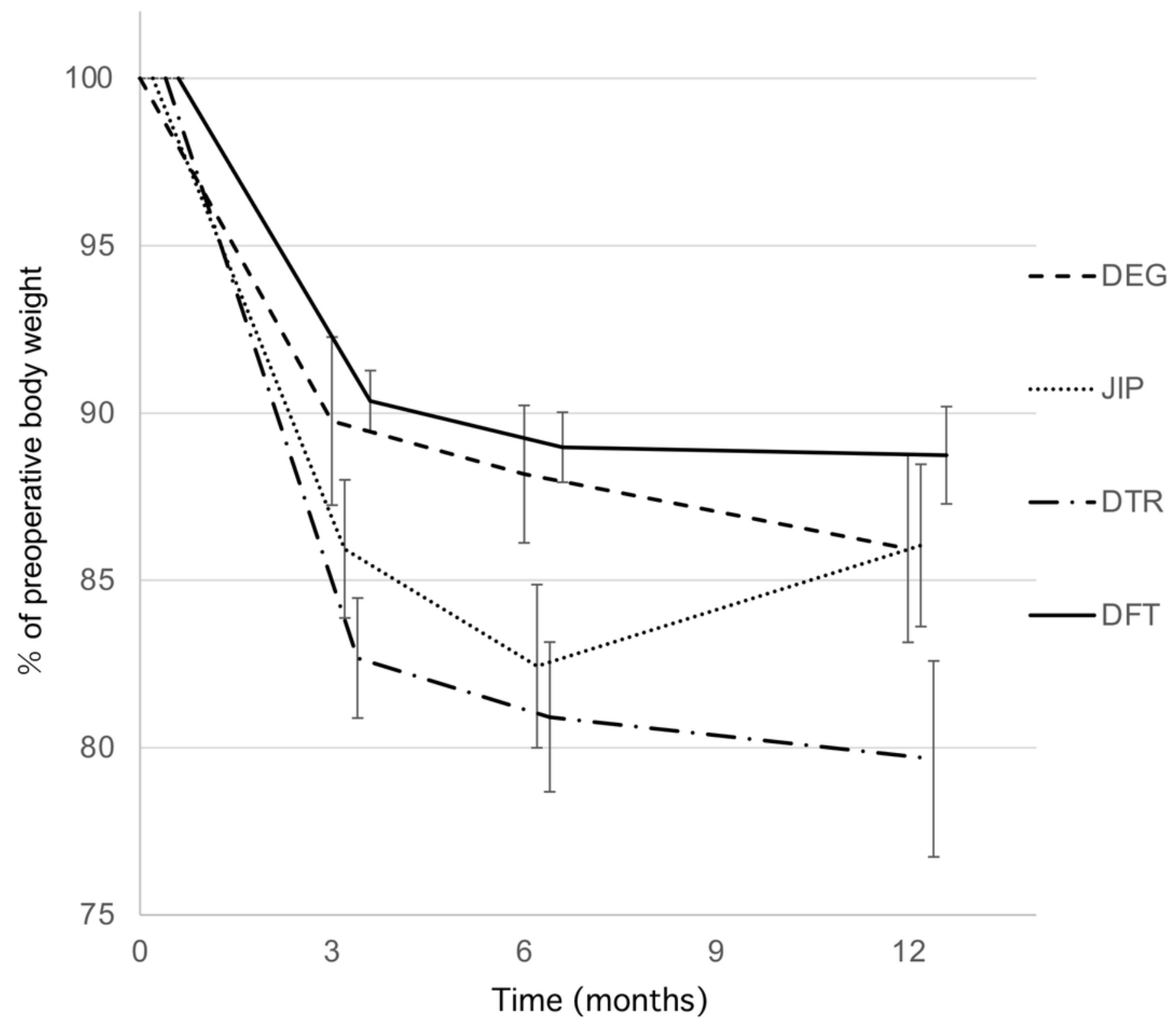

Figure 1

The percentage of post-operative body weight to the pre-operative. Data are expressed as mean \pm standard error. Body weight loss ratio of DFT was significantly better than DTR at 3-month, 6-month, and 12 -month after surgery $(p=0.001,0.003$, and 0.014 , respectively) 